\title{
Influence of growth conditions on microstructure and defects in diamond coatings grown by microwave plasma enhanced CVD
}

\author{
KALYAN SUNDAR PAL ${ }^{1,2, \dagger}$, SANDIP BYSAKH ${ }^{1, *}$, AWADESH KUMAR MALLIK ${ }^{1}$, \\ NANDADULAL DANDAPAT $^{1}$, SOMESWAR DATTA ${ }^{1}$ and BICHITRA K GUHA ${ }^{2}$ \\ ${ }^{1}$ Central Glass \& Ceramic Research Institute, Kolkata 700 032, West Bengal, India \\ ${ }^{2}$ Department of Physics, Indian Institute of Engineering Science and Technology, Howrah 711 103, India \\ †Present address: National Test House (ER), Kolkata 700 091, India
}

MS received 2 September 2014; revised 9 December 2014

\begin{abstract}
Diamond coatings were grown on $\mathrm{SiO}_{2} / \mathrm{Si}$ substrate under various process conditions by microwave plasma chemical vapour deposition (MPCVD) using $\mathrm{CH}_{4} / \mathrm{H}_{2}$ gas mixture. In this paper, we present a microstructural study to elucidate on the growth mechanism and evolution of defects, viz., strain, dislocations, stacking faults, twins and non-diamond impurities in diamond coatings grown under different process conditions. Transmission electron microscopy (TEM), X-ray diffraction (XRD) and Raman spectroscopy were used to characterize the diamond coatings. It has been shown that our new approach of prolonged substrate pre-treatment under hydrogen plasma yielded a new growth sequence that the $\mathrm{SiO}_{2}$ layer on the $\mathrm{Si}$ substrate was first reduced to yield $\mathrm{Si}$ layer of $\sim 150 \mathrm{~nm}$ thickness before diamond was allowed to grow under $\mathrm{CH}_{4}-\mathrm{H}_{2}$ plasma, created subsequently. It has also been shown that $\mathrm{Si}$ and $\mathrm{O}$ as impurity from the substrate hinders the initial diamond growth to yield non-diamond phases. It is being suggested that the crystal defects like twins, stacking faults, dislocations in the diamond grains and dislocations in the intermediate Si layer are generated due to the development of non-uniform stresses during diamond growth at high temperature.
\end{abstract}

Keywords. Microwave plasma CVD; polycrystalline diamond; transmission electron microscopy; microstructure; defects.

\section{Introduction}

Over the last few decades, chemical vapour deposition (CVD) grown diamond has been found to be suitable for wide range of applications like thermal management, ${ }^{1-3}$ tribological components, ${ }^{4,5}$ transmission windows, ${ }^{6,7}$ surface acoustic wave (SAW) devices, ${ }^{8}$ micro- and nano-electromechanical devices, ${ }^{9}$ etc. They have been reported to contain various kinds of impurities like graphitic inclusions, amorphous carbon, non-diamond impurities, structural defects like dislocations, twins, point defects, etc. The densities of such defects have been found to be controlled by CVD growth parameters, viz., flow rate of hydrogen, percentage of methane and substrate temperature. ${ }^{10,11}$ The density of crystal defects and the amount of non-diamond phases present in the diamond coating determine its shade, ranging from black to grey towards white and hence its suitability to different applications. Diamond coatings appearing dark under optical microscope contain high density of defects and can be used only for tribological applications and cannot be used as an optical component in instruments, e.g., gyrotron windows, X-ray optics, infrared spectrometer, etc. ${ }^{12}$ For suitable optical applications, CVD grown diamond needs to be free of crystal defects. It is now known that 'whiteness' and

\footnotetext{
*Author for correspondence (sbysakh@cgcri.res.in)
}

'darkness' of diamond can be manipulated by suitably varying the deposition parameters. ${ }^{10,13}$ Polycrystalline diamond (PCD) coatings grown by the microwave plasma enhanced chemical vapour deposition (MWPECVD) technique is used as window material for millimetre wave transmission in gyrotron tubes. Such material can be grown by the CVD technique into different grades like thermal grade, mechanical grade, optical grade, electronic grade. ${ }^{14,15}$ Essentially, better the quality of the PCD, less is the concentration of the defects mentioned above and more is the whitish appearance of the PCD wafer. For example, it has already been reported that for a given methane concentration, when the flow rate of hydrogen is increased, the appearance of diamond coating changes gradually from black to grey to white. ${ }^{10,16}$

There has been a significant number of reports during the last two decades on microstructural characterization of CVD grown diamond coatings. These studies were focused on defect analysis, ${ }^{17-19}$ presence of impurities,${ }^{20}$ microstructure of the interfaces and the dependence of the nature of defects on experimental growth parameters. ${ }^{21}$ There are also a few important reports ${ }^{22,23}$ elucidating on layer growth sequence during the initial stages of growth on substrate and reporting the formation of a SiC layer just before the actual growth of diamond crystals.

In the present work diamond coatings were grown by microwave plasma CVD under different experimental 
conditions by varying the microwave power, chamber pressure, methane:hydrogen ratio and flow rates. The purpose of the whole work was to grow good quality diamond coating by the MWPECVD technique, study the effect of varying the deposition process parameters on coating quality and microstructure and minimize the internal defects of diamond coating by optimization of the various deposition parameters. The objective of the present paper is to draw a comparison on the microstructure of diamond coatings of two different optical qualities, i.e., grey-scale appearances, e.g., dark and light, which were obtained for diamond coatings grown under two different process conditions. Analytical and high-resolution transmission electron microscopy (TEM) has been used as a primary tool for characterizing coating microstructure and crystal defects. An attempt has been made to corroborate the effect of growth conditions on microstructure in terms of the presence of various types of crystal defects, as well as their population density and elucidate on the mechanism of nucleation and growth of diamond grains in the coating. In addition, we present the result of our new approach of prolonged substrate pre-cleaning under $\mathrm{H}^{+}$plasma.

\section{Experimental}

\subsection{Materials and methods}

Diamond coatings were grown by using $915 \mathrm{MHz}$ MWPECVD system (DIAMOTEK 1800, Lambda Technologies Inc.). The detailed description of reactor geometry and the effect of process parameters on uniformity of temperature over the substrate were discussed in our previous work. ${ }^{24}$ Firstly, the as-received silicon wafers with $1-\mu \mathrm{m}$-thick oxide layer were seeded with detonation nano-diamond (DND). The DND seeded substrates were loaded into the MPCVD chamber with the help of computer-controlled automated loading mechanism. The chamber was then evacuated to $10^{-3}$ Torr by scroll type roughing pump (BOC Edwards XDS $35 i)$ and high vacuum $\left(10^{-7}\right.$ Torr $)$ was achieved by running turbo-molecular pump (BOC Edwards). Subsequently, hydrogen gas was bled into the system through mass flow controller at $500 \mathrm{sccm}$. The pressure was set to 5 Torr by adjusting the butterfly valve (MKS Type 153). Once the desired hydrogen flow rate and pressure were achieved, low power $1500 \mathrm{~W}$ plasma was struck by igniting the cathode filament. By choosing correct combination of the probe, short and stage positions, plasma was formed over the top of substrate wafer, in a symmetrical hemispherical shape.
Due care was taken to avoid errant plasma that might form at wrong locations like, bottom of the substrate stage or somewhere on the chamber wall. Ramping of microwave power from 1500 to $9000 \mathrm{~W}$ and ramping of chamber pressure from 5 to 110 Torr were programmed over a period of $1 \mathrm{~h}$. At first, only hydrogen gas was introduced into the chamber with a flow rate of $600 \mathrm{sccm}$ during ramping of microwave power from 1500 to $5000 \mathrm{~W}$ and ramping of pressure from 5 to 70 Torr so that only hydrogen plasma was formed initially and the same was maintained up to $7000 \mathrm{~W}$ power and chamber pressure of 100 Torr for 20 min in order to reduce the surface oxide on substrate. Subsequent to this substrate pre-treatment by $\mathrm{H}^{+}$plasma, methane was introduced inside the chamber so as to initiate the diamond growth. Subsequently, deposition was allowed to proceed with the pre-programmed CVD diamond deposition recipe.

A large number of diamond coatings were grown by varying the process parameters, viz., pressure, microwave power, methane-hydrogen flow rates and ratio and position of substrate with respect to probe and short. It was found that methane-hydrogen flow rates primarily determined the optical quality of the grown diamond coating. The best one (white) among these coatings and one of intermediate quality (grey) were chosen for comparative study. Table 1 compares two different process conditions for which the diamond coatings of two different qualities were obtained.

\subsection{Characterization of diamond films}

The crystalline phases and growth orientation of the diamond coatings were studied by X-ray diffractometry (XRD, PW 1710, Philips Research Laboratory, Eindhoven, The Netherlands) with $\mathrm{Cu}-\mathrm{K} \alpha$ radiation $(45 \mathrm{kV}, 35 \mathrm{~mA})$. In addition, the quality of the diamond coatings were examined by microRaman spectroscopy (HR-800, Horiba Jobin Yvon, Japan) with $10 \mathrm{~mW}$ laser power and $50 \mu \mathrm{m}$ spot size.

In order to carry out TEM studies, diamond coatings were grown up to a thickness of about 20-30 $\mu \mathrm{m}$. The coatings were then peeled off the Si substrate. For obtaining TEM specimen of the nucleation side, the free-standing diamond coatings were argon ion polished from the top surface, i.e., from the growth side. In order to investigate internal microstructure, specimen from within the thickness of the free-standing PCD coating was obtained by argon ion polishing from both sides till perforation. Cross-section specimens were obtained by cutting pieces of rectangular shape

Table 1. Experimental conditions for diamond coatings of two different qualities.

\begin{tabular}{|c|c|c|c|c|c|c|c|c|c|}
\hline $\begin{array}{l}\text { Sample } \\
\text { name }\end{array}$ & Quality & $\begin{array}{l}\text { Methane } \\
\text { flow rate } \\
(\mathrm{sccm})\end{array}$ & $\begin{array}{l}\text { Hydrogen } \\
\text { flow rate } \\
(\mathrm{sccm})\end{array}$ & $\begin{array}{c}\text { Substrate } \\
\text { temperature } \\
\left({ }^{\circ} \mathrm{C}\right)\end{array}$ & $\begin{array}{c}\text { Pressure } \\
\text { (Torr) }\end{array}$ & $\begin{array}{l}\text { Microwave } \\
\text { power } \\
\text { (W) }\end{array}$ & $\begin{array}{c}\text { Probe } \\
(\mathrm{cm})\end{array}$ & $\begin{array}{l}\text { Short } \\
(\mathrm{cm})\end{array}$ & $\begin{array}{l}\text { Stage } \\
(\mathrm{cm})\end{array}$ \\
\hline S1 & Grey & 25 & 850 & $960-1050$ & 118 & 9300 & 8.8 & 58.3 & 0.3 \\
\hline $\mathrm{S} 2$ & White & 25 & 1000 & $985-1065$ & 110 & 9000 & 8.7 & 58.2 & 0.5 \\
\hline
\end{tabular}


of about $2.6 \mathrm{~mm} \times 10 \mathrm{~mm}$, from the $\mathrm{Si}$ wafer along with adhered diamond coatings with the help of Buehler highspeed diamond saw (Buehler Inc., USA). Two such pieces were then sandwiched with GATAN G-1 epoxy (Gatan Inc., USA) to join the diamond coatings face to face. This sandwiched piece was then ground on emery paper to be given the shape of a rod of $2.6 \mathrm{~mm}$ diameter and $10 \mathrm{~mm}$ length. This rod was then jammed with GATAN G-1 epoxy (Gatan Inc., USA) inside a stainless-steel tube of $2.7 \mathrm{~mm}$ inner diameter and $3 \mathrm{~mm}$ outer diameter. Slices of about $200 \mu \mathrm{m}$ thickness were then cut from this mounted specimen with the help of Buehler Isomet Low-speed precision diamondsaw (Buehler Inc., USA). These slices were then ground on diamond paper to a thickness of about $70 \mu \mathrm{m}$ and subsequently argon ion-polished till electron transparency was obtained.

\section{Results and discussions}

\subsection{Confirmation of diamond formation by X-ray diffraction and Raman spectroscopy analysis}

The representative X-ray diffractograms (XRD) obtained from the as-grown diamond coating of samples S1 and S2 are shown in figure $1 \mathrm{a}$ and $\mathrm{b}$, respectively. The diffractograms clearly show the presence of (111), (220), (311) peaks of cubic diamond phase confirming its formation in both samples. However, the XRD pattern of diamond coating sample S2 showed a relatively high intensity, and sharp diffraction peak for (111) planes, whereas (220) peak intensity dominated in case of sample $\mathrm{S} 1$. These results suggest that for the sample S1 the growth of diamond grains took place preferentially along the [220] crystallographic direction, whereas, it is the [111] direction in case of S2 diamond coating. This means that under lower hydrogen flow rate 220 texture is preferred while a higher flow rate prefers 111 texture in the diamond coating.

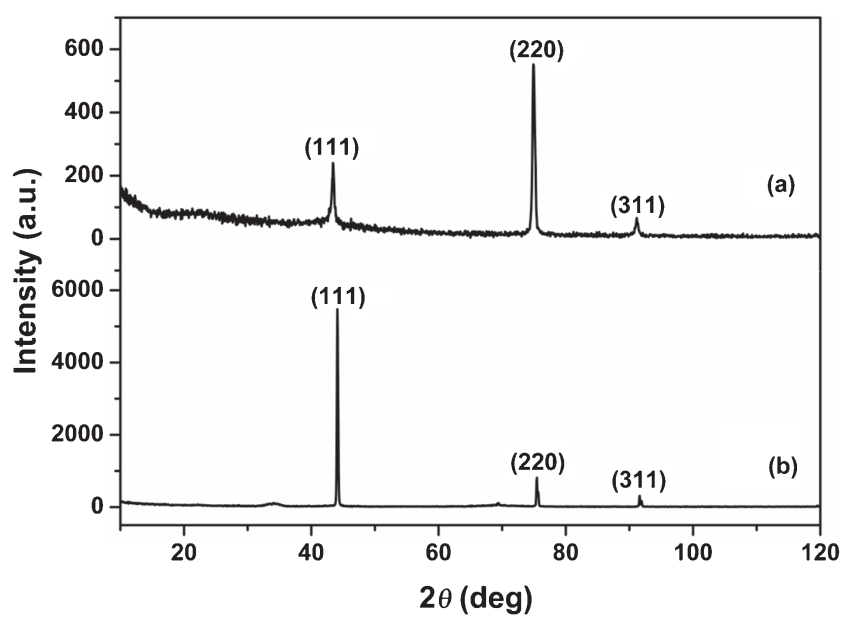

Figure 1. XRD results of the as-grown diamond coating for sample (a) S1 and (b) S2.
Further, a closer look into figure 1a reveals a slight broadening of all the diffraction peaks and a small hump in the $15^{\circ}<2 \theta<25^{\circ}$ range. The peak broadening hints at the presence of strain and defects within the diamond grains while the hump signifies the presence of amorphous carbon.

The crystalline quality of the diamond coatings was investigated by micro-Raman spectroscopy. Figure $2 \mathrm{a}$ and $\mathrm{b}$ shows the Raman shift at 1330.624 and $1330.538 \mathrm{~cm}^{-1}$ for samples $\mathrm{S} 1$ and $\mathrm{S} 2$, respectively. In case of sample $\mathrm{S} 2$, there are no other peaks or hump corresponding to non-diamond phases and therefore signifies a good quality. On the other hand, in case of sample S1, the signature of amorphous carbon is the band in the range of $1500-1700 \mathrm{~cm}^{-1}$ as shown in figure $2 \mathrm{~b}$. In addition, the noisy background and its rising intensity profile suggest highly luminescent character $^{25}$ of the coating. This can be attributed to the presence of crystal defects like vacancy, dislocations, stacking faults, etc. These micro-Raman spectroscopy results support the findings from the XRD analysis, as discussed above.

\subsection{Microstructural study of nucleation side of diamond coating}

In order to investigate the nature of defects and non-diamond phases, the diamond coating samples were characterized by TEM. Analytical TEM results of nucleation side of the diamond coating, S1, are presented in figure 3. The lowmagnification TEM micrograph of the area is presented in figure $3 \mathrm{a}$. The image shows a microstructure comprising of equi-axed granular features of 20-40 $\mu \mathrm{m}$ size separated by a low-contrast phase, filling the inter-granular spacing. This finding is therefore suggestive of grain coalescence during subsequent growth to yield a coating microstructure comprising of densely packed diamond crystals, as will be presented later in this paper. A closer look into the thinned electrontransparent regions of several granules revealed that they are

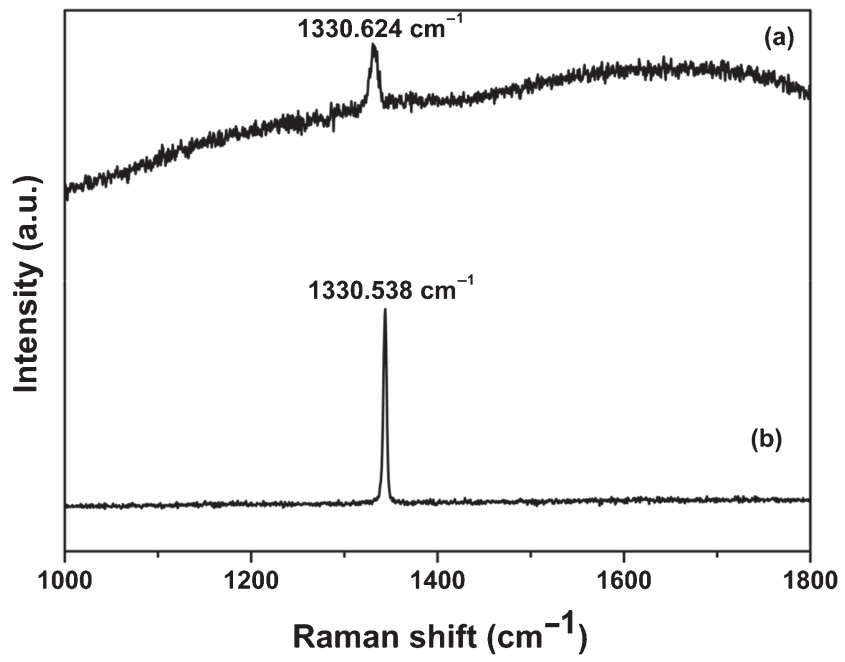

Figure 2. Raman spectroscopy of the as-grown diamond coating for sample (a) S1 and (b) S2. 


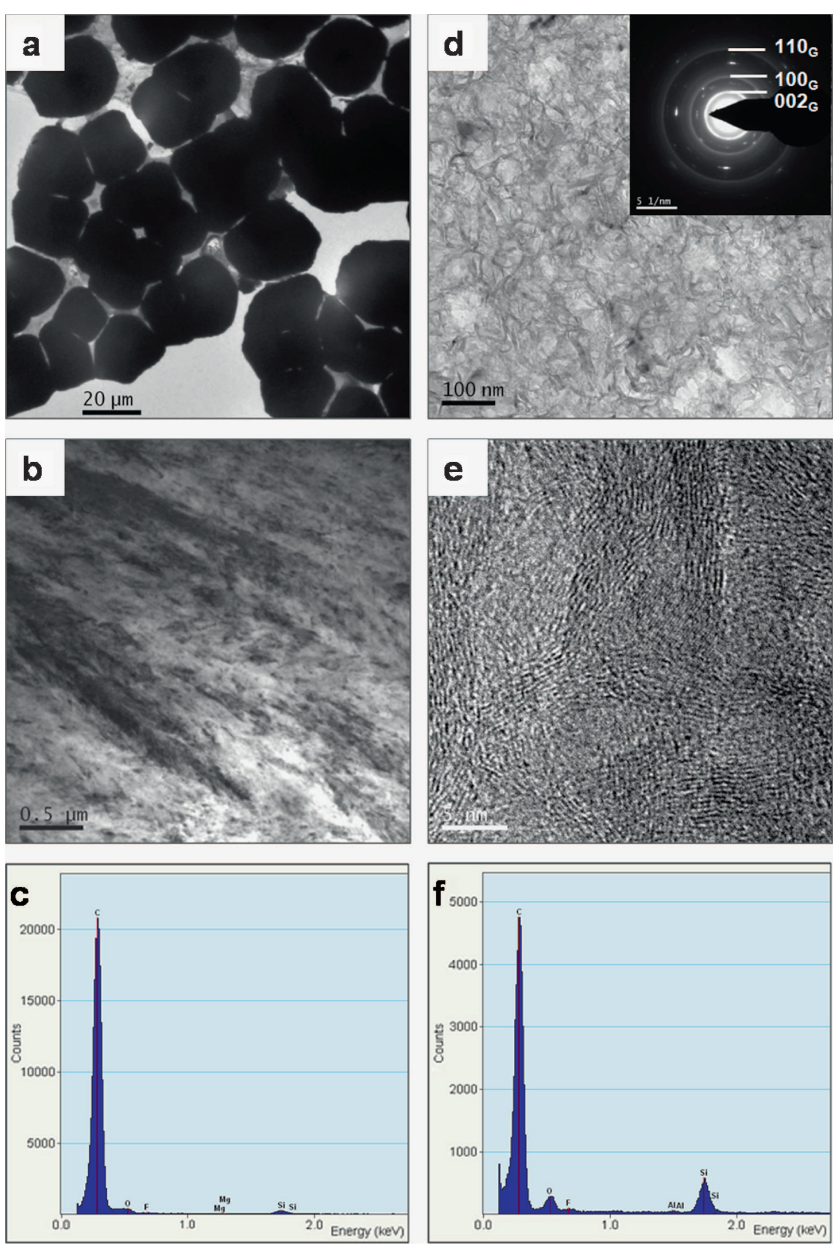

Figure 3. TEM results from nucleation side of diamond coating of sample S1 just on the surface of Si wafer. (a) Low-magnification bright-field TEM image showing $\sim 20-50 \mu \mathrm{m}$ size granules. (b) Bright-field TEM image showing directional growth morphology of crystalline phase within a grain. (c) EDX spectrum from one granule shows large $\mathrm{C}$ peak and a small peak of Si. (d) Bright-field TEM image of the inter-granular area showing graphitic morphology, (e) corresponding HRTEM image shows bent crystal planes typical of graphitic layers. (f) EDX spectrum from intergranular region shows relatively higher amounts of $\mathrm{O}$ and $\mathrm{Si}$ compared with their amounts within the granules.

composed of nano-crystals dispersed in amorphous carbon matrix, rather than each granule being an individual single crystal as shown in figure $3 \mathrm{~b}$. Formation of nanocrystalline diamond within graphitic amorphous phase during the initial growth stages has already been reported in literature. ${ }^{26,27}$ In our work this has also been confirmed by EDS analysis that the 'granules' are primarily made of carbon with small amounts of oxygen $(\sim 0.5$ at $\%)$ and $\mathrm{Si}(\sim 0.15$ at $\%)$ present as impurity. The relevant EDX spectrum presented in figure $3 \mathrm{c}$ shows the peaks of $\mathrm{O}$ and $\mathrm{Si}$. The bright-field TEM (BF-TEM) image of the inter-granular phase is shown in figure $3 \mathrm{~d}$, inset being the characteristic selected area diffraction pattern (SADP) of this region. The diffraction rings are due to graphitic carbon, while the spots in the
SADP can be indexed as reflections of orthorhombic diamond [PDF no. 79-1472]. Figure 3e is the HRTEM image of this intergranular region showing 'bent' lattice image typical of graphitic carbon ${ }^{28}$ co-existing with an amorphous phase. The characteristic EDX spectrum in figure $3 f$ from this intergranular region showed slightly higher amounts of $\mathrm{Si}(\sim 1.5$ at $\%)$ and $\mathrm{O}(\sim 2.0$ at $\%)$ as impurities as compared with the $\sim 20-50 \mu \mathrm{m}$ size granules.

The results for the nucleation side discussed above suggest that upon generating $\mathrm{CH}_{4}-\mathrm{H}_{2}$ plasma in the MPCVD chamber, at first the carbon-rich amorphous coating starts growing over the substrate. There is a role of atomic $\mathrm{H}$ and ionic $\mathrm{H}^{+}$in hydrogen plasma that cause etching of non-diamond phases. The presence of $\mathrm{Si}$ and $\mathrm{O}$ in the 'intergranular' regions and also within the granules at relatively lower concentration clearly indicates that $\mathrm{H}_{2}$ plasma reacted with the $\mathrm{SiO}_{2} / \mathrm{Si}$ substrate during the initial stages of growth of diamond coating. Such reaction with substrate and consequent Si contamination and nucleation of diamond within the initially grown amorphous phase is in agreement with an earlier report in the literature. ${ }^{26}$ As the diamond coating grows in thickness, the concentration of the impurities from the substrate is much reduced as found within the granules.

The coating that grew over the initial contaminated layer seems to be in the form of clusters containing largely of graphitic carbon within which diamond nanocrystals started nucleating. This finding in the present work suggests that the radial growth morphology comprising of clusters of nanocrystals within the micron-size granular features is formed centering a DND seed nanocrystal. Subsequently, as the growth progressed, the diamond grains grew bigger within the amorphous carbon under the $\mathrm{CH}_{4}-\mathrm{H}_{2}$ plasma as the impurity concentrations are minimized.

\subsection{Comparative TEM study of microstructure of diamond coatings}

Within its thickness, the free-standing diamond coating sample S1, showed well-developed crystalline microstructure. Figure $4 \mathrm{a}$ is an intermediate magnification BF-TEM image from within the coating thickness. The image shows a generally grainy microstructure with sharp grain boundaries. Therefore, the diamond grains grew and coalesced completely and no inter-granular non-diamond phases are seen. However, the grains exhibit defect morphology and variations in dynamic contrast are seen due to non-uniform strain in the grain. A higher-magnification BF-TEM image in figure $4 \mathrm{~b}$ shows a triple-junction grain boundary. The grains exhibit the presence of non-uniform strain contrast and dislocations. This indicates that the diamond grains developed internal stresses to nucleate defects within the crystal lattice during growth from vapour phase. The microstructure in the BF-TEM image in figure $4 \mathrm{a}$ also shows varieties of other morphological defects like twinning and stacking faults. The HRTEM image in figure $4 \mathrm{c}$ was taken from an area showing twinning of diamond crystals. The mirror image relationship between the crystal lattice planes of the 

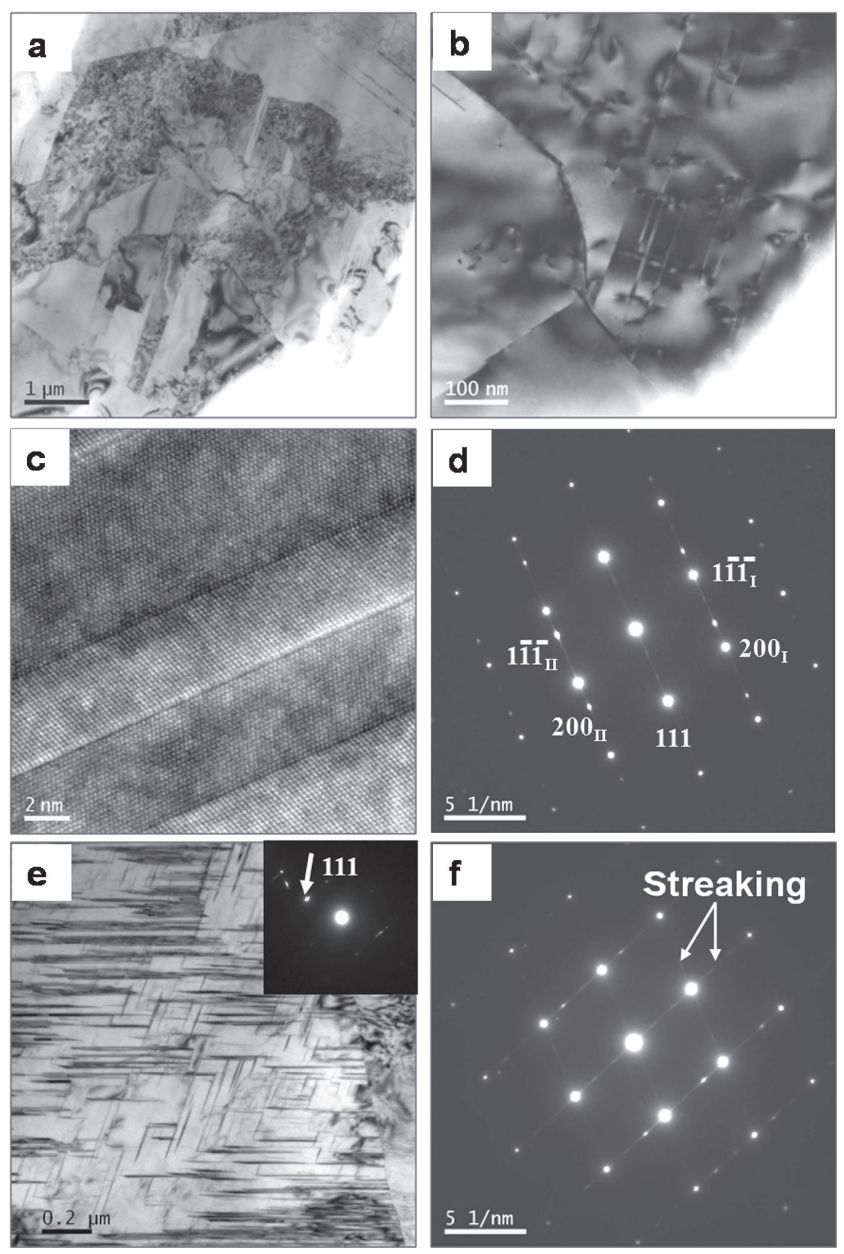

Figure 4. TEM results from within the thickness of diamond coating of sample S1. (a) Low-magnification BF image showing diamond grains with defect morphology in general. (b) Highermagnification BF-TEM image showing a grain containing line and planar defects. (c) HRTEM image showing twin. (d) SAD from c showing evidence of twinning. (e) BF morphology of a grain with high density of stacking faults. (f) SADP showing streaking along the two 111 directions due to SF on these 111 planes, presence of nano-crystals within granular features and as well as intergranular regions.

adjacent twin plates can be clearly observed. The characteristic SADP from this area clearly shows the evidence of twinning on (111) planes. The patterns contributed from the two twin variants are marked in figure $4 \mathrm{~d}$. The morphology of a diamond grain with a high density of stacking faults is revealed in the BF-TEM image in figure 4e. Inset shows the diffraction condition for imaging the stacking faults. The characteristic SADP of this grain shows streaking along the $\langle 111\rangle^{*}$ reciprocal lattice vectors as indicated by arrows in figure 4f. This suggests that the stacking faults are lying on 111 planes of the diamond cubic structure. The density of dislocations varied from grain to grain. Figure 5a and $\mathrm{b}$ are the bright-field and weak-beam dark-field (WBDF) TEM images, respectively, of a diamond grain with a relatively high density of dislocations. Thus, sample $\mathrm{S} 1$ grown at
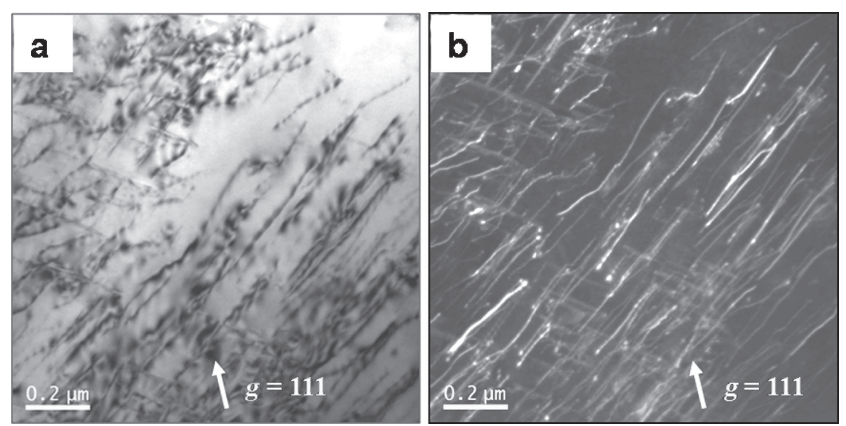

Figure 5. TEM results from within the grain of diamond coating of sample S1. (a) Bright-field TEM image showing a diamond grain with high dislocation density. (b) Weak-beam dark-field (WBDF) TEM image showing individual dislocations clearly discernible.

relatively low hydrogen flow rate $(850 \mathrm{sccm})$ contains various types of defects, mostly dislocations, stacking faults, twins and even amorphous regions.

The varied density of dislocations, stacking faults and twins from grain to grain suggests that the individual grains are stressed to different extent, i.e., non-uniform stresses developed in the coating during growth. It is also possible that for a given experimental growth condition which promotes crystallographic defects, some grains may be growing with crystallographic orientations that favour nucleation of stacking faults and twins to relax the stress, while other grains grow under stress generating dislocations during growth. There are reports ${ }^{29,30}$ in the literature that at high temperatures in the vicinity of $1000^{\circ} \mathrm{C}$, diamond does deform and dislocation movements take place under stress. Therefore, deformation of CVD diamond coating during growth at high temperature to generate dislocations and planar defects may not be ruled out.

On the other hand, the diamond coating sample S2, which was grown at a higher hydrogen gas flow rate $(1000 \mathrm{sccm})$, is composed of well-defined large crystallites with significantly reduced population of defects. Majority of the grains, specially, large crystallites of 10-20 $\mu \mathrm{m}$ size, has been found to be defect free. Only a few diamond grains contained small number of dislocations while no stacking faults could be observed within any crystal. Figure 6a showed BF-TEM image of a defect free diamond grain. The absence of dislocations and other defects was also confirmed by BF-TEM imaging at different diffraction conditions by tilting the specimen over a wide range of angular orientations with respect to the incident electron beam. The corresponding SAD pattern along $[01 \overline{1}]$ zone in figure $6 \mathrm{c}$ clearly shows no streaking of spots and therefore suggests the absence of any planar defects. However, some diamond grains exhibited twinned morphology. Figure 6b shows BF-TEM image of one such grain containing twins near a triple junction. The corresponding $\mathrm{SAD}$ pattern in figure $6 \mathrm{~d}$ shows the evidence of multiple twins.

At this point it will be appropriate to make a mention of the contribution by Lee and co-workers ${ }^{31}$ in their review paper, where the various work on nucleation phenomenon and growth mechanism of diamond films by CVD have been 


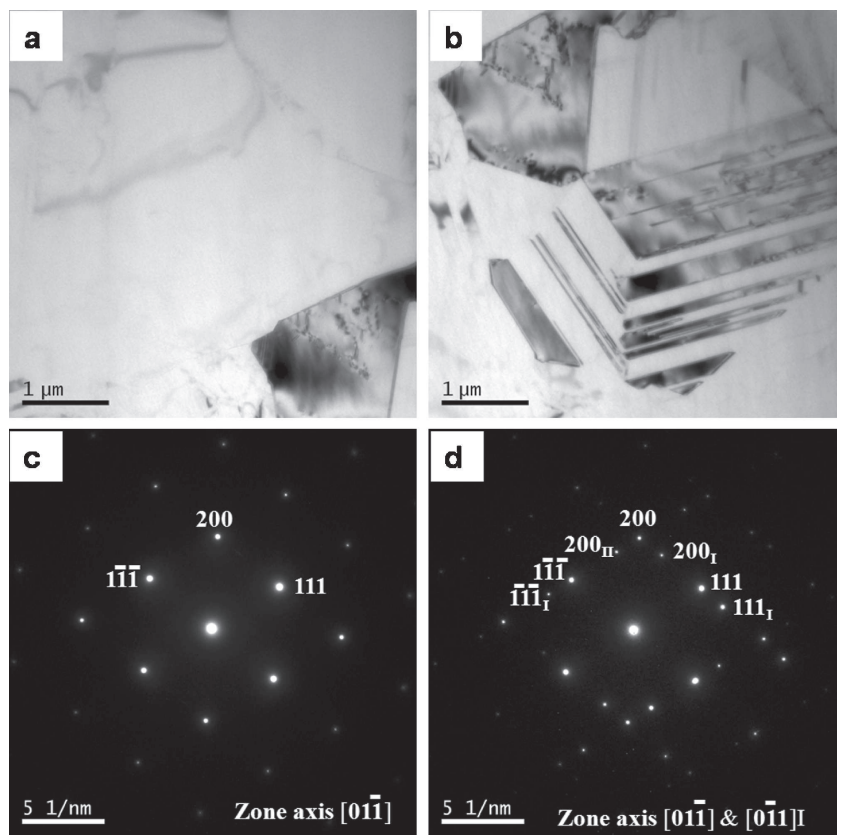

Figure 6. TEM results from within the thickness of diamond coating of sample S2. (a) BF image showing defect-free diamond grains. (b) BF-TEM image showing a grain containing line defects and also showing triple junction twin. (c) SAD from corresponding to a showing the defect free morphology. (d) SAD from corresponding to $\mathbf{b}$ showing the evidence of twinning.

discussed in detail. From all the research work discussed in that review, it has been established that both atomic $\mathrm{H}$ and $\mathrm{H}^{+}$ions in the plasma play the most important role in preferentially etching the deposited hydrocarbons, stabilizing the $\mathrm{sp}^{3}$ bonding in diamond nuclei and maintaining clean facets of growing diamond crystals. This phenomenon forms the prime basis for the evolutionary selection principle of diamond nucleation and growth. In addition, there is also a theoretical study ${ }^{32}$ involving calculation using density functional theory (DFT) to predict atomic structure of nucleating diamond. The paper discusses the elementary steps of nucleating carbon seeds for the next layer growth through hydrocarbon adsorption and subsequent surface carbon cluster formation to provide an atomistic insight into twin formation during the CVD diamond growth. This insight corroborates with the results from our present work in that during growth the diamond crystals are quite likely to develop twins, which were found in all samples including S1 and S2 observed under TEM.

\subsection{Cross-section TEM study of the coating-substrate interface}

In order to elucidate upon the layer growth mechanism during diamond growth on $\mathrm{SiO}_{2} / \mathrm{Si}$ substrate, TEM of the diamond coating-on-substrate cross-section was carried out for sample S2. The bright-field TEM image in figure 7a shows a distinct intermediate layer of about $150 \mathrm{~nm}$ thickness between the Si-oxide layer and the diamond coating. The SAD pattern from the native oxide layer that was present over the as-received Si substrate shows diffraction halo ring, suggesting its amorphous nature. Diffraction evidence from the intermediate layer at different specimen tilt angles with respect to the electron beam in the TEM revealed that this intermediate layer is actually a single crystal of Si produced by H-Plasma reduction of $\mathrm{SiO}_{2}$ layer. The presence of occasional faceted pores and the dislocations present within this layer revealed by the bright-field (figure 7a) and weakbeam dark-field image (figure 7b), respectively, further confirms this intermediate layer to be a single grain or crystal of $\mathrm{Si}$ as observed within the limited field of view in TEM imaging. However, this same single crystal of Si may not be covering the entire substrate, if this intermediate $\mathrm{Si}$ layer is polycrystalline. The corresponding inset shows single crystal SAD pattern along [013] DC zone. The diamond cubic structure of this intermediate layer is confirmed by the weak 200 reflection in the SAD zone pattern and absence of 200 reflection under two-beam condition for 400 reflection. Thus, the intermediate Si layer appears to be composed of large single crystals of Si laterally grown over the substrate surface oxide $\left(\mathrm{SiO}_{2}\right)$ layer. The elemental distribution maps for Carbon, oxygen and Silicon obtained by STEM-EDS spectrum imaging technique are presented in figure $7 \mathrm{c}-\mathrm{e}$, respectively. The maps provide the direct evidence that the intermediate layer formed between the substrate surface oxide and the diamond coating is of Si. Nano-probe EDS composition analysis suggested that the composition of the intermediate layer is nearly pure $\mathrm{Si}$, except only a few percent of oxygen being present. The results of TEM observation discussed above are therefore, in agreement with earlier work, ${ }^{33,34}$ which also reported growth of diamond grains directly on $\mathrm{Si}$ without the formation of the reaction layer of $\mathrm{SiC}$ below diamond coating.

The results described above help one understand the evolution of microstructure in diamond coating and layer growth sequence by microwave plasma enhanced CVD. Crosssection TEM study provides the direct evidence of an intermediate layer of crystalline $\mathrm{Si}$. The presence of this intermediate Si layer suggests that before starting diamond growth, the initially generated hydrogen plasma reacts with the surface oxide $\left(\mathrm{SiO}_{2}\right)$ layer on the $\mathrm{Si}$ substrate. As a result, the oxide layer gets reduced to yield an intermediate $\mathrm{Si}$ layer composed of large crystallites containing dislocations. Cross-sectional scanning electron microscopy (SEM) study of diamond coating on $\mathrm{Si}$ substrate was published earlier by other research group ${ }^{19}$ to report columnar growth of diamond, but in their case, the formation of such intermediate layer composed of large Si crystals was not observed. Our observation of the formation of large crystalline Si layer containing dislocations is different from the findings by other workers, ${ }^{21-23}$ who reported the formation of $\mathrm{SiC}$ islands at the substrate-coating interface.

In this context, it is important to discuss our new approach of prolonged substrate pre-treatment under $\mathrm{H}^{+}$plasma, as has been adopted in the present work. The present technique 


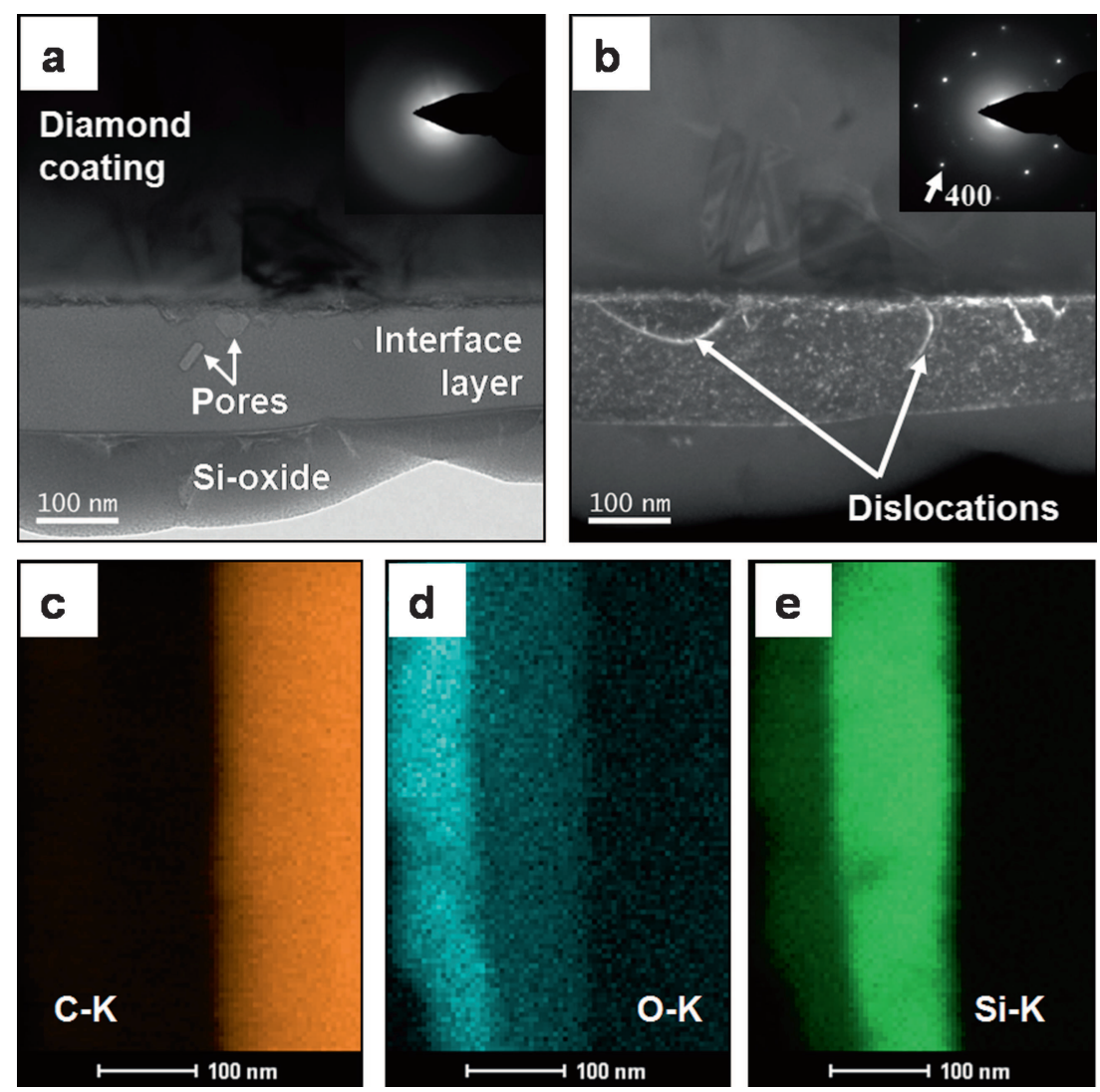

Figure 7. Transmission electron microscopy results showing interface layer between diamond coating and Si substrate. (a) Bright-field TEM image shows interface layer of $\sim 150 \mathrm{~nm}$ thickness. (b) Weak-beam dark-field TEM image of the crystalline layer reveals dislocations. (c-e) The elemental maps for carbon, oxygen and silicon obtained by collecting $\mathrm{K} \alpha$ characteristic X-ray emission in the STEM-EDS spectrum imaging technique. The map in $\mathbf{e}$ is the direct evidence of Si layer formation on the Si-oxide layer of the $\mathrm{SiO}_{2} / \mathrm{Si}$ substrate.

of substrate pre-cleaning is entirely different from techniques reported in numerous earlier publications by other researchers. ${ }^{9}, 16,22,23$ Most of these publications report substrate pre-treatment involving mechanical polishing, ultrasonic treatment and seeding with nano-diamond suspension and mechanical embedding of natural diamond particles in the substrate surface. However, none of these works have reported the use of $\mathrm{H}^{+}$plasma in order to remove substrate surface oxide. Although there is one report of substartate pretreatment by $\mathrm{H}^{+}$plasma, ${ }^{21}$ the purpose was to remove any hydrocarbon on substrate surface. Our approach of prolonged $\mathrm{H}^{+}$plasma pre-treatment of substrate surface is entirely different from other's work in that the purpose was to remove the surface oxide through process of reduction by $\mathrm{H}^{+}$plasma. The intermediate Si layer is a new outcome of our approach.

The nucleation of dislocations in the intermediate Si layer is most likely due to the development of thermal stress during growth of diamond grains. However, the presence of oxygen impurity might also have played a role in nucleating dislocations. In our work, the presence of dislocations in the intermediate $\mathrm{Si}$ layer along the interface is probably being reported for the first time. Microstructural evolution with respect to defect, stacking faults, micro-twining, dislocations of diamond coating has been observed by detail TEM study. In plan-view TEM study, it is observed that within thickness of the diamond coating the density of dislocations, stacking faults and micro-twins are varied from sample $\mathrm{S} 1$ to $\mathrm{S} 2$ due to variation of the experimental growth conditions. In sample S2, defect free diamond grains were observed for certain optimized process parameters like high $\mathrm{H}_{2}$ flow rate with respect to low methane concentration, moderate microwave power and chamber pressure. Thus the TEM study in this work played an essential role to rationalize the origin and evolution of defects in diamond coatings. It was also discussed in terms of possible improvement of quality of diamond coating by TEM study which is supported by Raman spectroscopy as well as XRD.

In summary, the main differences of our work from the reported ones are the following:

(1) A new approach of prolonged substrate pre-treatment under $\mathrm{H}^{+}$plasma to remove surface oxide i.e., $\mathrm{SiO}_{2}$, on $\mathrm{Si}$ substrate, before initiating diamond growth under $\mathrm{CH}_{4}-\mathrm{H}_{2}$ gas-mixture plasma. 
(2) As an outcome of our new approach for substrate pre-cleaning under $\mathrm{H}^{+}$plasma, we have presented the direct evidence of $\sim 150 \mathrm{~nm}$ thick, laterally large, crystalline Si intermediate layer formed between substrate and diamond coating, rather than several reports on $\mathrm{SiC}$ intermediate layer formation. In addition, the direct proof of dislocation nucleated within this intermediate Si layer due to stresses developed due to diamond growth, has also been provided.

(3) We have given direct evidence of the presence of all types of crystal defects, as well as reduction in their population through optimization in deposition process parameters.

(4) In our present work, is has been shown that TEM study can play essential role to investigate crystal defects and microstructure in diamond coatings and corroborate their evolution with growth conditions, which need to be optimized for growing diamond coatings of improved crystal quality.

\section{Conclusions}

1. Significant improvement of the diamond quality and an almost defect free diamond coating has been grown by combination of optimized process parameters like high hydrogen flow rates, moderate microwave power and chamber pressure of microwave plasma CVD.

2. Cross-sectional TEM study provides the outcome of our newly adopted process of prolonged substrate pretreatment under hydrogen plasma. The direct evidence of formation of an intermediate layer of crystalline $\mathrm{Si}$ is presented. This is suggestive of the fact that before starting diamond growth, substrate pre-treatment under $\mathrm{H}^{+}$plasma reduced the surface oxide $\left(\mathrm{SiO}_{2}\right)$ to yield the intermediate Si layer.

3. The presence of dislocations in the intermediate $\mathrm{Si}$ layer is most likely due to the development of thermal stress during growth of diamond grains and possibly being reported for the first time.

4. The dislocations, twins and stacking faults observed in diamond grains possibly formed due to stresses at high temperature during the growth of diamond, while dislocation movements under non-uniform stresses may not be ruled out.

\section{Acknowledgements}

We gratefully thank Council for Scientific and Industrial Research for financial support for carrying out the present work. We also like to thank the Director, Central Glass and Ceramic Research Institute, for the permission to publish this work.

\section{References}

1. Coe S E and Sussmann R S 2000 Diam. Relat. Mater. 91726
2. Brown W D, Beera R A, Naseem H A and Malshe A P 1996 Surf. Coat. Technol. 86698

3. Willander M, Friesel M, Wahab Q U and Straumal B $2006 \mathrm{~J}$. Mater. Sci.: Mater. Electron. 171

4. Mallik A K, Shivashankar S A and Biswas S K 2009 Sadhana 34811

5. Silva F J G, Fernandes A J S, Costa F M, Teixeira V, Baptista A P M and Pereira E 2003 Wear 255846

6. Thumm M 2001 Diam. Relat. Mater. 101692

7. Brandon J R, Coe S E, Sussmann R S, Sakamoto K, Sporl R, Heidinger R and Hanks S 2001 Fusion Eng. Des. 53553

8. Liu J M, Xia Y B, Wang L J, Su Q F, Zhao P, Xu R, Peng H Y and Shi W M 2006 Trans. Nonferrous Met. SOC China 16 s298

9. Sumant A V, Auciello O, Yuan H C, Ma Z, Carpick R W and Mancini D C 2009 Proc. SPIE $7318731817-1$

10. Nistor L, Landuyt J V and Ralchenko V 1999 Phys. Status Solidi (a) $\mathbf{1 7 4} 5$

11. Aleksandrov V D and Selskaya I V 2003 Inorg. Mater. 39455

12. Yang J X, Duan X F, Lu F X, Li C M, Zuo T C and Wang F L 2005 Diam. Relat. Mater. 141583

13. Ralchenko V G, Pleuler E, Lu F X, Sovyk D N, Bolshakov A P, Guo S B, Tang W Z, Gontar I V, Khomich A A, Zavedeev E V and Konov V I 2012 Diam. Relat. Mater. 23172

14. May P W 2000 Philos Trans. R. Soc. Lond. A 358473

15. Butler J E and Sumant A V 2008 Chem. Vapour Depos. 14145

16. Ralchenko V, Sychov I, Vlasov I, Vlasov A, Konov V, Khomich A and Voronina S 1999 Diam. Relat. Mater. 8189

17. Delclos S, Dorignac D, Phillipp F, Silva F and Gicquel A 1998 Diam. Relat. Mater. 7222

18. Delclos S, Dorignac D, Phillipp F, Silva F and Gicquel A 2000 Diam. Relat. Mater. 9346

19. Nistor L C, Landuyt J V, Ralchenko V G, Obraztsova E D and Smolin A A 1997 Diam. Relat. Mater. 6159

20. Yin L W, Zou Z D, Li M S, Sun D S, Liu Y X and Hao Z Y 2000 Diam. Relat. Mater. 92006

21. Wittorf D, Jager W, Dieker C, Floter A and Guttler H 2000 Diam. Relat. Mater. 91696

22. Waite M M and Shah S I 1992 Appl. Phys. Lett. 602344

23. Kato Y, Goto M, Sato R, Yamada K, Koga A, Teii K, Srey C and Tanaka S 2011 Surf. Coat. Technol. 206990

24. Mallik A K, Pal K S, Dandapat N, Guha B K, Datta S and Basu D 2012 Diam. Relat. Mater. 3053

25. Filik J 2005 Spectrosc. Europe 1710

26. Lin T, Loh K P, Wee A T S, Shen Z X, Lin J, Lai C H, Gao Q J and Zhang T J 2000 Diam. Relat. Mater. 91703

27. Nistor L, Ralchenko V, Vlasov I, Khomich A, Khmelnitskii R, Potapov P and Landuyt J V 2001 Phys. Status Solidi (a) 186 207

28. Ajeian B, Beckey H D, Maas A and Nitschke U 1975 Appl. Phys. A: Mater. Sci. Process. 6111

29. Comins J D, Daniel R D, Erasmus R M and Brookes E J 2000 Diam. Relat. Mater. 91115

30. Brookes C A 1979 The properties of diamond Field JE (Ed.) (London Academic Press) p 383

31. Lee S T, Lin Z and Jiang X 1999 Mater. Sci. Eng. R 25123

32. Butler J E and Oleynik I 2008 Phil. Trans. R. Soc. A 366 295

33. Jiang X and Jia C L 1995 Appl. Phys. Lett. 6791197

34. Jia C L, Urban K and Jiang X 1995 Phys. Rev. B 525164 